\title{
FLOATING-DISK PARYLENE MICRO CHECK VALVE
}

\author{
Po-Jui Chen and Yu-Chong Tai \\ Micromachining Laboratory, California Institute of Technology \\ 1200 E California Blvd M/C 136-93, Pasadena, CA 91125 USA \\ Tel: +1-626-395-2254 Fax: +1-626-584-9104 E-mail: pjchen@mems.caltech.edu
}

\begin{abstract}
A novel micro check valve which has nearly ideal fluidic shunting behaviors is presented. Featuring a parylene-based floating disk, this surface-micromachined check valve ultimately realizes both zero forward cracking pressure and zero reverse leakage in fluidic operations. Two different floating disk designs have been implemented to demonstrate functionality of the microvalve. Experimental data of underwater testing successfully show that in-channel floating-disk valves in both designs have great fluidic performance close to an ideal check valve, except the additional fluidic resistance in the order of $10^{13} \mathrm{~N}-\mathrm{s} / \mathrm{m}^{5}$ based on dimensions of the fabricated devices. Their pressure loading limit have been confirmed to be higher than $300 \mathrm{kPa}$ without water leakage. This type of micro check valve is believed to have great use of flow control in integrated microfluidics and lab-on-a-chip applications.
\end{abstract}

\section{INTRODUCTION}

Micro check valves has been engineered for almost 20 years in micro-electro-mechanical systems (MEMS)/microfluidics research [1][2]. Researchers have developed various kinds of micro check valves during this period to pursue a check valve close to an ideal valve, particularly having the following three characteristics in terms of flow control: (1) zero reverse leakage, (2) zero forward cracking pressure, and (3) zero forward fluidic resistance. Even though the last criteria is theoretically impossible for general flow operations, the first two could be achieved while they have never been realized in a single valve. This fact leaves a big room for microvalve improvement.

Currently, all existing micro check valves, regardless of being surface-micromachined or bulk-micromachined, have either forward cracking pressure or reverse leakage problems illustrated in Fig. 1(a) [3]. As a result, these microvalves are usually recognized as either "normallyclosed" or "normally-open" check valves related to their fluidic shunting performance. This imperfect bidirectional valve behavior definitely limit the practical use of microvalves as a flow control component in integrated microfluidic systems, specifically in miniature pressure/flow rate operations. In this work, therefore, we have developed a surface-micromachined check valve with refined valve performance in order to generate more potentials for microvalves to be utilized in real applications. By using a novel floating-disk design, the proposed microvalve is the first micro check valve so far as known that realizes both zero reverse leakage and zero forward cracking pressure in a single valve illustrated in Fig. 1(b) as a nearly ideal check valve. Moreover, the structural material of this thin-film valve is selected to be parylene, a biocompatible polymeric material which facilitates the valve completely suitable for lab-on-a-chip systems in life science research.

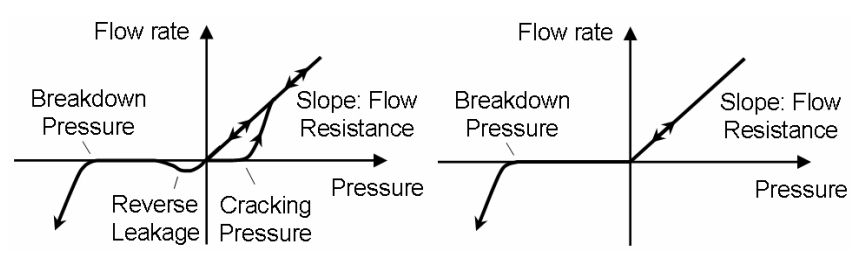

(a)

(b)

Figure 1. Micro check valve behaviors: (a) Conventional valve; (b) Valve developed in this work.

\section{DESIGN}

In order to eliminate forward cracking pressure and reverse leakage, the fluidic shunting mechanism of a micro check valve requires a mechanical moving part which does not have any lagging response with respect to injected hydraulic pressure. Accordingly, we have developed a microvalve incorporating an unclamped "floating disk" as the key moving element as shown in Fig. 2(a). The actuation principle is analogous to the free-plate design of the Knowles SiSonic ${ }^{\mathrm{TM}}$ MEMS microphone [4]. Because the disk diaphragm is not connected/tethered to any structure, it acts as a free plate in simply supported condition in mechanics and can move out-of-plane immediately by applied with a minimal loading. As confined in between a top cap and a bottom seat, the disk diaphragm floats inside and switches its position to be in contact with top cap or bottom seat depending on applied pressures. Consequently, by placing appropriate orifices in disk/cap/seat combination, this valve configuration enables conducting and blocking flows with minimum resistance and hysteresis, which eventually solves the forward cracking pressure and reverse leakage problems. Two designs (Design A: solid disk and valve cap with peripheral orifices; Design B: disk with central orifice and valve cap with peripheral orifices) in Fig. 2(b) regarding different orifice locations were implemented to accomplish the floating-disk valve behaviors with different directions of flow injection. Tolerance of in-plane disk diaphragm movement inside the spacing between the cap and the seat needs to be especially considered to prevent accidental overlap of the orifices causing operation failure 
in practice. Parylene C (poly-para-xylylene C) was selected as a thin-film structural material because of its ease of processing, CMOS/MEMS processes compatibility, chemical inertness, and biocompatibility (USP Class VI grade). All these advantages favor the microvalve in onchip microfluidic applications. Gravitational effect on the parylene floating disk was insignificant to influence the nearly ideal fluidic shunting behavior based on the fact of its small mass (in the order of $10^{-13} \mathrm{~g}$ ) at the desired device scale compared to pressure loadings from microflows of general interest $(>1 \mathrm{kPa})$.

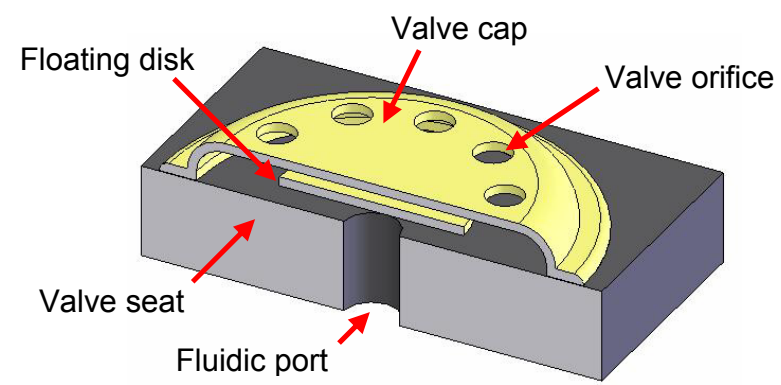

(a)
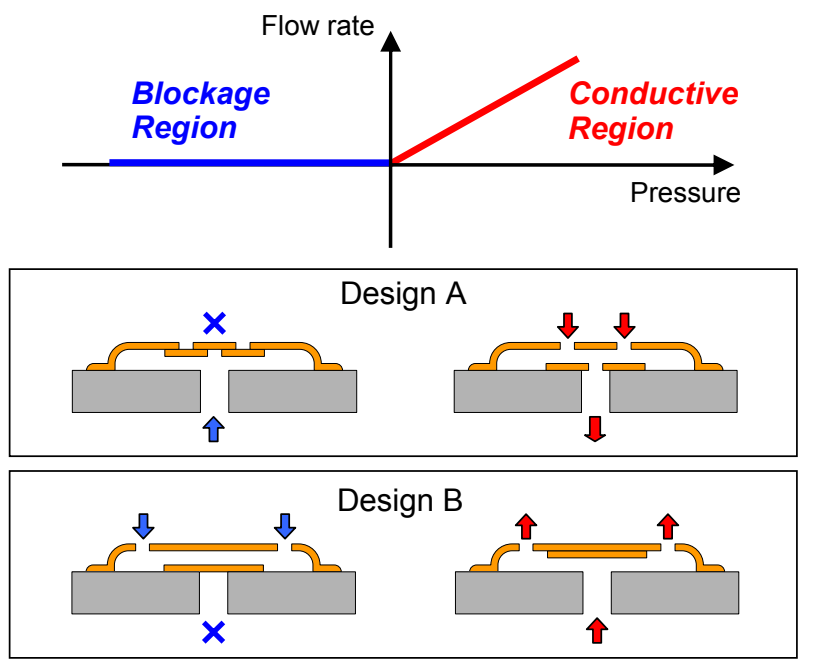

(b)

Figure 2. Floating-disk valve design: (a) Conceptual schematic; (b) Operation regions realized with two designs of valve disk/cap/seat combination that conduct (red arrows on the right side) and block (blue arrows on the left side) flows in different directions of flow injection.

\section{FABRICATION}

The fabrication process is illustrated in Fig. 3 started with growing a $2 \mu \mathrm{m}$ wet oxide on a standard silicon wafer. After oxide patterning the backside of wafer was etched by using deep-reactive-ion-etching (DRIE) until leaving about $50 \mu \mathrm{m}$ silicon membrane. This step was to define the fluidic coupling port on the silicon valve seat. On the frontside of wafer a parylene multi-layer surfacemicromachining technology was applied to deposit/pattern three parylene $\mathrm{C}$ structural layers (disk diaphragm: $1.5 \mu \mathrm{m}$; valve cap: $3 \mu \mathrm{m}$; fluidic channel: $5 \mu \mathrm{m}$, all in thickness). Parylene patterning was performed by using oxygen plasma etching with photoresist as the mask material. During the processes the sacrificial photoresist was hard-baked at $120{ }^{\circ} \mathrm{C}$ if applicable for edge-smoothening and degassing purposes. All parylene $\mathrm{C}$ layers were roughened by a short period of oxygen plasma treatment to reduce stiction effect in device operations afterwards. Furthermore, in order to enhance the device robustness in terms of high pressure capacity, the adhesion of parylene/silicon interface at valve cap and channel layers was physically promoted by roughening the silicon surface using gas-phase $\mathrm{XeF}_{2}$ etching before parylene deposition [5]. After the surfacemicromachining processes, the remaining silicon membrane was etched away from backside of the wafer to create through-holes on the valve seat. Not only did these through-holes create fluid access to the in-channel microvalves but make the photoresist exposed and able to be stripped with acetone for final device release.
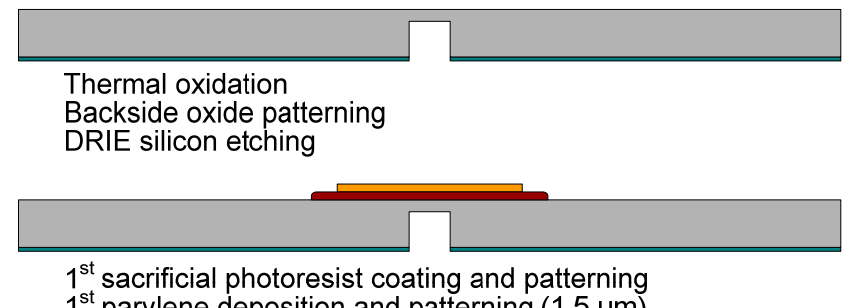

$1^{\text {st }}$ parylene deposition and patterning $(1.5 \mu \mathrm{m})$
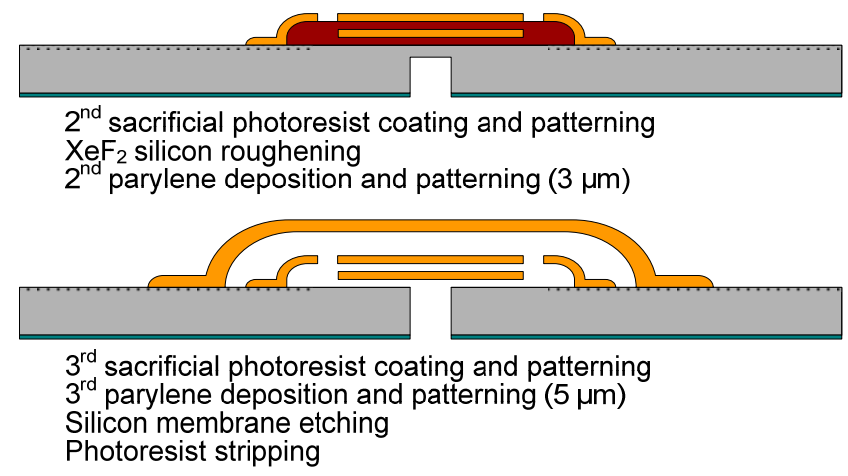

Silicon $\square \mathrm{SiO}_{2} \quad \square$ Parylene Photoresist

Figure 3. Fabrication process flow.

Although using photoresist as a sacrificial material is convenient in the surface-micromachining processes, it may bring other difficulties to the floating-disk valve fabrication. In principle, the fabricated free-plate disk diaphragm needs to be highly flat for reliable valve behaviors, while constructing flat sacrificial photoresist base for following disk layer deposition/patterning is challenging by spin coating techniques. Here a thin $(\sim 1.5 \mu \mathrm{m})$ layer of photoresist was coated and hard-baked to flatten the sacrificial base as much as possible. The profile scan of the photoresist base as shown in Fig. 4 indicates a sufficiently flat surface was obtained at the central region. The 
peripheral region of the base, however, became bumpy due to photoresist reflow after baking. It is obvious that the flat valve disk diaphragm must be defined at the central flat region of the photoresist base, which generates in-plane clearance and resulting movement of the floating disk inside the valve cap and seat. This phenomenon needs to be taken care of by appropriate geometrical designs of the microvalve in order not to influence the fluidic shunting behaviors of them.

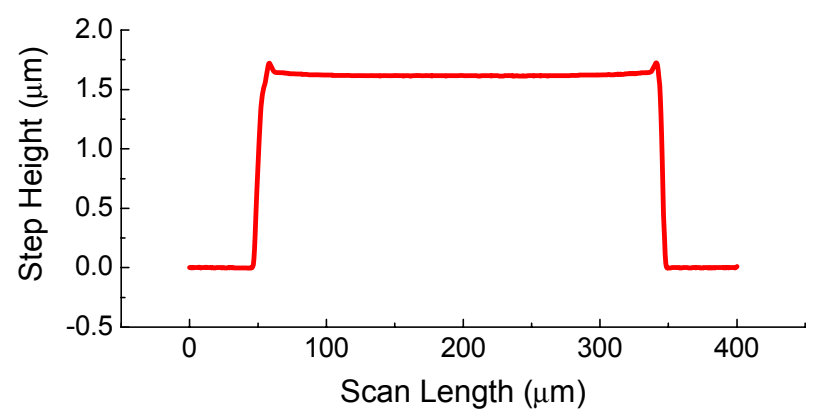

Figure 4. Profile scan of the actual sacrificial photoresist base for floating disk diaphragm deposition/patterning.

An important issue to the floating-disk check valve is that, after device release, the fabricated microvalves should be always kept in liquid solutions to avoid stiction of the disk diaphragm. A disk diaphragm can realize the floating function if there is no tethering structure connecting to it. It means, on the other hand, the disk can not have any support to resist the attraction caused by surface tension to other surfaces during drying, which ends with device failure. The microvalve could possibly be rejuvenated by performing disk-popping pressurization after drying, but many efforts need to be done toward a reliable process [6]. In this work, instead, the fabricated devices were immersed in liquids to avoid occurrence of the air/liquid/solid interface. In fact, this action retrieves more design flexibility on the valve cap without stiction concerns so that smaller out-of-plane spacing of the valve cap/disk/seat could be obtained for better fluidic shunting behaviors. Fig. 5 shows a fabricated floating-disk parylene micro check valve after drying, and the underwater in-channel parylene microvalves are shown in Fig. 6. The disk diaphragms were off from the center as expected due to their freedom of in-plane movement.

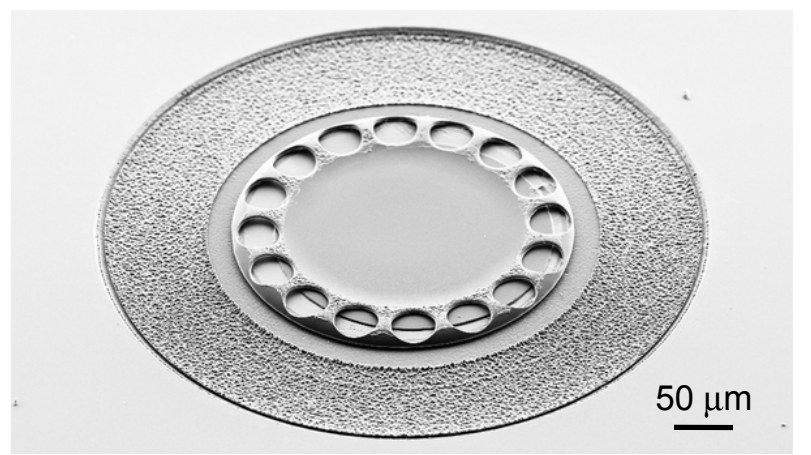

Figure 5. SEM image of fabricated floating-disk check valve (in Design A). Stiction happened after device drying.
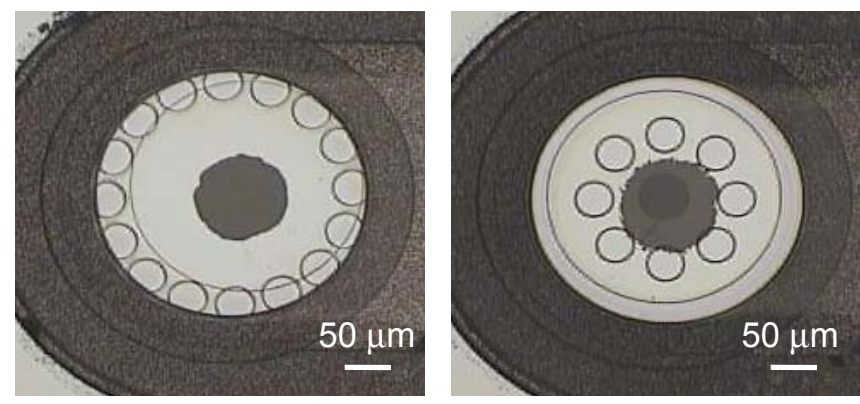

Figure 6. Micrographs of in-channel floating-disk parylene micro check valves in Design A (left) and Design B (right) immersed in water (top view).

\section{TESTING AND DISCUSSION}

Fabricated on-chip in-channel microvalves were tested using the fluidic setup as illustrated in Fig. 7 derived from previous work [3]. A customized testing jig was used to provide a macroscale/microscale fluidic interface with diced chips so that microflows could be introduced to the devices through their backside fluidic access ports with assistance of regular tubing and fittings. Clean water served as the working fluid source. Pressure control of the injected water flow in the resolution of approximately $70 \mathrm{~Pa}(\sim 0.01 \mathrm{psi})$ was achieved using combination of a commercial off-chip pressure regulator and pressure gauge. Flow rate measurement was performed by recording the marching velocity of the water column front in the capillary tube. In order to maintain the microvalves functional, the testing jig was immersed in water as well as air bubbles in the jig and gas columns in the front capillary tube were completely removed prior to valve characterization.

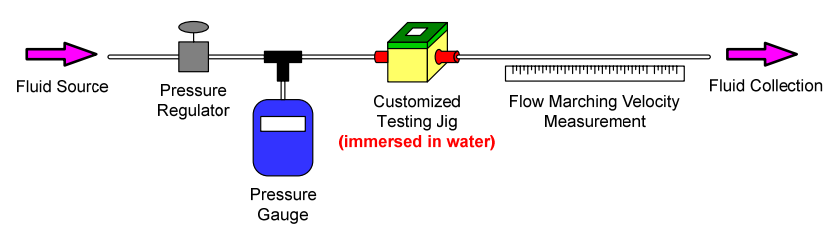

Figure 7. Schematic of fluidic setup for device testing.

The pressure/flow rate testing data of the devices are shown in Fig. 7. Hollow microchannels without encapsulating any micro check valve were also tested to give a reference to the valved channel characterization. Other than the first-order fluidic resistance effect, the second-order flow-induced channel deformation effect was also considered in the pressure/flow rate analysis to fit the data points more accurately [7][8]. It is evident from the plot that, for both valve designs (Design $A$ and Design $B$ ), no cracking pressure to the forward flow or reverse leakage to the backward flow of the fluid was observed in the valved channels with pressure loadings down to $700 \mathrm{~Pa}$ ( $\sim 0.1 \mathrm{psi})$ from the testing setup. This result implies that the floatingdisk micro check valves were able to shunt the water microflow with minimum pressure loadings. The insertion of the microvalves did generate additional fluidic resistance, 
which was $1.25 \times 10^{13} \mathrm{~N}-\mathrm{s} / \mathrm{m}^{5}(\sim 0.03 \mathrm{psi}-\mathrm{min} / \mu \mathrm{L})$ in Design A and $2.09 \times 10^{13} \mathrm{~N}-\mathrm{s} / \mathrm{m}^{5}(\sim 0.05 \mathrm{psi}-\mathrm{min} / \mu \mathrm{L})$ in Design B, approximately $16 \%$ and $26 \%$ increase to that of an individual microchannel. The fluidic resistance of the valve in Design $\mathrm{A}$ is higher than Design $\mathrm{B}$ because longer equivalent fluidic channel and more complicated flow pathway was created from those valve disk/cap structures. In backflow operations, although the parylene floating disk diaphragms were experienced some plastic deformation after operated with large pressure loadings $(>300 \mathrm{kPa})$, they were still confined at the original location and the associated microvalves stayed leakage-proof. However, device failure happened at the encapsulating microchannels. Several devices were tested and the breakdown pressure was found to be in a range of 317-393 $\mathrm{kPa}(\sim 46-57 \mathrm{psi})$, at which microchannels were always seriously delaminated from the surface so water leakage was coming out from the channels. This fact resulted from insufficient adhesion at parylene/ silicon interface even after silicon roughening treatment. A more robust channel that can withstand higher injection pressure loadings will be necessary in the future in order to investigate the real breakdown pressure with failure mode directly in the floating-disk microvalves. Table I summarizes some important characteristics of the state-ofthe-art surface-micromachined in-channel micro check valves for comparison.

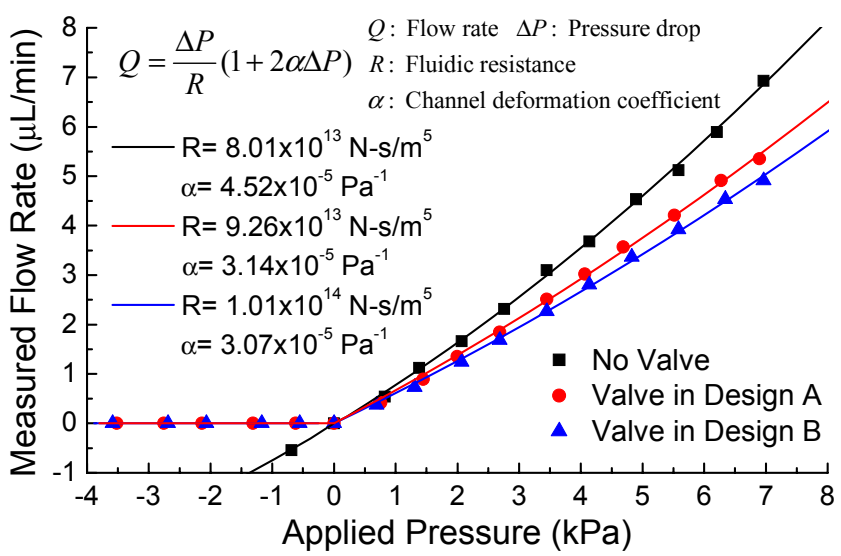

Figure 8. Device testing results with water as the working fluid. Individual microchannel was tested as reference.

Table I Comparison of in-channel micro check valves.

\begin{tabular}{|c|c|c|}
\hline & [3] & This work \\
\hline Configuration & $\begin{array}{c}\text { Tethered } \\
\text { diaphragm }\end{array}$ & $\begin{array}{c}\text { Floating } \\
\text { disk }\end{array}$ \\
\hline Occupied area & $\sim 10^{-7} \mathrm{~m}^{2}$ & $\sim 10^{-7} \mathrm{~m}^{2}$ \\
\hline Dead volume & $\begin{array}{l}\sim 1 \mathrm{~nL}\left(\mathrm{NO}^{*}\right) \\
\sim 3 \mathrm{~nL}\left(\mathrm{NC}^{*}\right)\end{array}$ & $\sim 1 \mathrm{~nL}$ \\
\hline Cracking pressure & $\begin{array}{l}<0.1 \mathrm{psi}\left(\mathrm{NO}^{*}\right) \\
\sim 0.2 \mathrm{psi}\left(\mathrm{NC}^{*}\right)\end{array}$ & $\sim 0$ \\
\hline $\begin{array}{l}\text { Pressure to stop } \\
\text { reverse leakage }\end{array}$ & $\begin{array}{c}1.5 \mathrm{psi}\left(\mathrm{NO}^{*}\right) \\
0\left(\mathrm{NC}^{*}\right) \\
\end{array}$ & $\sim 0$ \\
\hline Breakdown pressure & $>25 \mathrm{psi}$ & $>40 \mathrm{psi}$ \\
\hline
\end{tabular}

$* \mathrm{NO}=$ normally-open valve $\mathrm{NC}=$ normally-closed valve

\section{CONCLUSION}

A parylene-based floating-disk micro check valve has been successfully developed. By incorporating an unclamped free-plate disk diaphragm as the moving part, the microvalve can shunt fluids with minimum pressure loadings and ultimately achieves nearly ideal check valve behaviors such as zero forward cracking pressure and zero reverse leakage in microflow operations. The in-channel micro check valves in two different geometrical designs have been successfully fabricated by using a parylene multilayer surface-micromachining technology. Device characterization was demonstrated in underwater environment showing excellent fluidic shunting behaviors to water microflow. From the testing results along with biocompatible property of parylene material, the floatingdisk micro check valves have great capability of being integrated into microfluidic systems for life science research.

\section{ACKNOWLEDGEMENTS}

This work was supported in part by the Engineering Research Centers Program of the National Science Foundation under NSF Award Number EEC-0310723. The authors especially thank Mr. Jason Shih for his valuable comments on flow measurements and analysis, and $\mathrm{Mr}$. Trevor Roper for his fabrication assistance.

\section{REFERENCES}

[1] S. Shoji and M. Esashi, "Microflow Devices and Systems," Journal of Micromechanics and Microengineering, 4(4), pp. 157-171, 1994.

[2] K.W. Oh and C.H. Ahn, "A Review of Microvalves," Journal of Micromechanics and Microengineering, 16(5), pp. R13-R39, 2006.

[3] P.-J. Chen, D.C. Rodger, E. Meng, M.S. Humayun, and Y.-C. Tai, "Surface-Micromachined In-Channel Parylene Dual Valves for Unpowered Microflow Regulation," Proc. HiltonHead 2006 Workshop, Hilton Head Island, SC, USA, Jun. 4-8, 2006, pp. 205-208.

[4] P.V. Loeppert and S.B. Lee, "SiSonic ${ }^{\mathrm{TM}}$ - The First Commercialized MEMS microphone," Proc. HiltonHead 2006 Workshop, Hilton Head Island, SC, USA, Jun. 4-8, 2006, pp. 27-30.

[5] X. Q. Wang, Q. Lin, and Y.-C. Tai, "A Parylene Micro Check Valve," Proc. MEMS 1999 Conference, Orlando, FL, USA, Jan. 17-21, 1999, pp. 177-182.

[6] J. Xie, X. Yang, X. Q. Wang, and Y.-C. Tai, "Surface Micromachined Leakage Proof Parylene Check Valve," Proc. MEMS 2001 Conference, Interlaken, Switzerland, Jan. 21-25, 2001, pp. 539-542.

[7] J. Shih, J. Xie, and Y.-C. Tai, "Surface Micromachined and Integrated Capacitive Sensors for Microfluidic Applications," Proc. Transducers 2003 Conference, Boston, MA, USA, Jun. 8-12, 2003, pp. 388-391.

[8] T. Gervais, J. El-Ali, A. Günther, and K.F. Jensen, "FlowInduced Deformation of Shallow Microfluidic Channels," Lab on a Chip, 6(4), pp. 500-507, 2006. 